\title{
Monitoring human serum albumin cell cultures using surface plasmon resonance (SPR) spectroscopy
}

\author{
A. Henseleit, C. Pohl, Th. Bley, and E. Boschke \\ Institute of Food Technology and Bioprocess Engineering, Technische Universität Dresden, 01062 Dresden, \\ Germany
}

Correspondence to: A. Henseleit (anja.henseleit@tu-dresden.de)

Received: 23 October 2014 - Revised: 18 December 2014 - Accepted: 22 January 2015 - Published: 13 February 2015

\begin{abstract}
Continuously monitoring cell cultures is essential for both controlling critical parameters and improving understanding of key processes. An ideal technique in this context is surface plasmon resonance (SPR) spectroscopy, which essentially exploits changes in the angle of incident light that occur when molecules bind to a surface. It provides the ability to monitor real-time changes in small concentrations of various molecules, with no need for additional labels or sample preparation. Here we present an SPR-based immunoassay for monitoring concentrations of human serum albumin (HSA), and compare its sensitivity when used in conjunction with a Biacore platform and the cheaper, smaller ${ }^{\text {li }}$ SPR system. In conjunction with either system, the immunoassay can detect HSA (a hepatocyte viability marker) at concentrations typically present in three-dimensional hepatocyte cultures mimicking the liver used to evaluate effects of drug candidates before exposure to humans or animals.

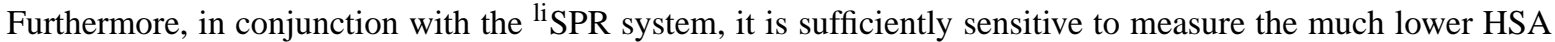
levels present in skin-hepatocyte co-cultures.
\end{abstract}

\section{Introduction}

Human serum albumin (HSA) is continuously synthesized by hepatocytes. The heart-shaped, $66.5 \mathrm{kDa}$ large protein is the most abundant plasma protein and is responsible, inter alia, for transporting toxins from the liver (Curry et al., 1999). The serum albumin concentration in blood (typically ca. $0.6 \mathrm{mM}$ ) can be used to evaluate the metabolic activity of mammals and diagnosing various diseases, e.g., liver or kidney diseases, infectious diseases and cancer (Yang et al., 2011). In clinical studies, HSA is also used to characterize specific activities of in vitro cultivated hepatocytes, and thus predict effects of pharmaceuticals. Notably, Wagner et al. monitored HSA levels, among other factors, to assess the viability of hepatocytes in liver and skin tissues co-cultivated on a multiorgan chip used to simulate processes in the human body (Wagner et al., 2013). Thus, relevant processes of a substance in the human body can be simulated. To measure the viability of the hepatocytes, they detected, among others, the HSA level during the cultivation period of 14 days. They showed that HSA was consumed by the skin tissue, and measured HSA concentrations ranged from 3.3 to $3.8 \mathrm{nM}$, far lower than concentrations in purely hepatocyte cultivations (88 to $170 \mathrm{nM})$. Thus, to monitor such cell-chip platforms, highly sensitive methods capable of detecting target proteins rapidly (ideally in real time) in minimal samples are required.

For such purposes, surface plasmon resonance (SPR) spectroscopy has highly attractive features, including capacities to continuously monitor multiple samples with minimal volumes highly sensitively (Henseleit et al., 2014). Using SPR, interactions between label-free target molecules and receptors can be detected in real time based on refractive index changes in an evanescent field arising at a metal-dielectric interface. Appropriate receptors for a target molecule are immobilized on the surface of a sensor providing such an interface and samples are injected in a microfluidic flow. Surface plasmons are then excited, usually by the Kretschmann configuration, in which a light beam of constant intensity and frequency is directed through a prism onto a gold surface and totally reflected at the metal-prism interface (Homola, 2008; Kretschmann and Raether, 1968). At a certain angle of incidence, the light transfers some of its energy to the electrons at the gold surface, thereby reducing the intensity of the re- 
flected light (Henseleit et al., 2011). When molecules bind to (or dissociate from) the surface, the refractive index and thus the angle at which the energy transfer occurs changes immediately. The change in intensity of the reflected light is detected by an optical unit and is proportional to the mass of the binding (or dissociating) molecules (Cass et al., 1998). The resulting shifts in the intensity minima are presented as a function of time in a sensorgram.

In the study presented here, we used a low-cost ${ }^{\text {li }}$ SPR system (capitalis technology $\mathrm{GmbH}$ ) and the well-established Biacore instrument (T100, GE Healthcare Europe $\mathrm{GmbH}$ ) for the specific and sensitive detection of HSA. Biacore instruments were the first commercially available optical SPRbased biosensors and are still widely used. However, they are costly, large and thus not ideal for point-of-care applications. In marked contrast, the liSPR instrument is a benchtop spectrometer, which affords high sensitivity and up to 180 sensing spots (Henseleit et al., 2011). We assessed the utility of SPR spectroscopy generally, and both the wellestablished Biacore platform and the ${ }^{\text {li }}$ SPR system specifically, for immunologically measuring HSA concentrations of in vitro hepatocyte cultures. For this purpose, biotinylated HSA-specific antibodies were bound to streptavidin immobilized on the two systems' sensor surfaces, to exploit the extreme strength of non-covalent streptavidin/biotin binding (dissociation constant, ca. $4 \times 10^{-14} \mathrm{M}$ ) (Cao et al., 2006; Holmberg et al., 2005; Peluso et al., 2003; Schneider et al., 2000).

\section{Materials and methods}

\subsection{Materials}

HSA-specific antibodies and streptavidin were purchased from Biomol GmbH (Germany). Biotinylated reference antibodies (BrdU-specific antibodies) were obtained from BioLegend, Inc. (USA). HSA, 11-mercaptoundecanoic acid and running buffer TBST (Tris buffered saline, with Tween ${ }^{\circledR}$ 20, pH 8.0) were purchased from Sigma-Aldrich Chemie GmbH (Germany). The amine coupling kit used to immobilize streptavidin (see below) was purchased from GE Healthcare Europe GmbH (Germany). All other chemicals were of analytical grade and obtained from VWR International GmbH (Germany).

\section{2 li SPR experiments}

The experiments carried out using the ${ }^{\text {li }}$ SPR system (capitalis technology $\mathrm{GmbH}$, Germany) were performed at $30^{\circ} \mathrm{C}$ with a flow rate of $5 \mu \mathrm{L} \mathrm{s}^{-1}$. The sensor surface of the system, a $50 \mathrm{~nm} \times 12 \mathrm{~mm} \times 3 \mathrm{~mm}$ gold layer, is illuminated by three selectable LEDs, each covering an area of $(9 \times 0.8) \mathrm{mm}^{2}$ (Henseleit et al., 2011; Mertig et al., 2009). A CCD camera with a spatial resolution of 1280 rows (spots) and an angledependent intensity distribution of 960 columns records the reflected light (Mertig et al., 2009). In this study, only the middle LED was used.

Levels of protein bound to the surface were measured in pixels, each corresponding to 41 resonance units (RU, $1 \mathrm{RU} \approx 1 \mathrm{pg} \mathrm{mm}^{-2}$ ) (Henseleit et al., 2014). The raw data were evaluated using Microsoft Excel.

\subsubsection{Preparation of the gold surface and SAM}

To prepare carboxylated self-assembled monolayers (SAM), the bare gold surfaces of liSPR sensor chips were first treated with UV/Ozone (UV/Ozone ProCleaner, NanoAndMore $\mathrm{GmbH}$, Germany) for $30 \mathrm{~min}$ and then rinsed with pure ethanol. The clean gold surfaces were immersed in $10 \mathrm{mM}$ 11-mercaptoundecanoic acid overnight at $30^{\circ} \mathrm{C}$, thoroughly rinsed sequentially with $\mathrm{ddH}_{2} \mathrm{O}$, ethanol, $\mathrm{ddH}_{2} \mathrm{O}, 100 \mathrm{mM}$ $\mathrm{HCl}, 50 \mathrm{mM} \mathrm{NaOH}, 0.5 \%(v / v) \mathrm{SDS}$, and $\mathrm{ddH}_{2} \mathrm{O}$, and then dried under a stream of nitrogen.

\subsubsection{Streptavidin $\mathrm{pH}$ scouting}

Prior to the covalent immobilization of the streptavidin, the optimal pH was identified by "scouting", as follows. Solutions of $3.6 \mu \mathrm{M}$ streptavidin in $10 \mathrm{mM}$ sodium acetate with $\mathrm{pH}$ values ranging from 3.5 to 5.5 were prepared, injected onto the carboxylated surface (prepared as described above) for $2.5 \mathrm{~min}$, and then the bound molecules were removed by sequential injections of $500 \mu \mathrm{L}$ of $100 \mathrm{mM} \mathrm{HCl}, 50 \mathrm{mM}$ $\mathrm{NaOH}, 0.5 \%(v / v)$ SDS, and $\mathrm{ddH}_{2} \mathrm{O}$.

\subsubsection{Immobilization of the antibodies}

For the immobilization purpose, $10 \mathrm{mM}$ of sodium acetate ( $\mathrm{pH} 4.5)$ were used as the running dielectric as well as for dilution of the molecules.

Before immobilizing the biotinylated antibodies, the streptavidin molecules were immobilized using the amine coupling kit mentioned above according to the manufacturer's instructions, as follows. The carboxylated surface was activated for $10 \mathrm{~min}$ by injecting EDC/NHS (400 mM 1ethyl-3-(3-(dimethyl amino)-propyl)carbodiimide/100 mM $\mathrm{N}$-hydroxysuccinimide). The activated surface was then incubated for $1 \mathrm{~h}$ with a $9.3 \mu \mathrm{M}$ solution of streptavidin, followed by $1 \mathrm{M}$ ethanolamine- $\mathrm{HCl}$ for $30 \mathrm{~min}$ to block the remaining active groups. This resulted in immobilization of streptavidin at surface densities equivalent to $3437 \mathrm{RU}$. A $10 \mathrm{mM}$ sodium acetate $(\mathrm{pH} 4.5)$ was used as the running dielectric and for diluting the molecules during these immobilization procedures. Finally, $1.4 \mu \mathrm{M}$ of biotinylated antibody was immobilized on the streptavidin layer by incubation for $1 \mathrm{~h}$ in TBST. All signals presented here are raw signal measurements minus signals recorded from injections with a reference surface prepared by immobilizing biotinylated BrdU-specific antibodies on the streptavidin layer using the same strategy. 


\subsubsection{HSA measurement}

To assess the sensitivity of the ${ }^{\text {li }}$ SPR system, a series of solutions of HSA in TBST with concentrations ranging from 0.4 to $192.5 \mathrm{nM}$ were injected onto the antibody-modified surface for $10 \mathrm{~min}$. The level of binding was determined by measuring SPR signals directly after this association phase, which was followed by injecting a TBST buffer for about $3 \mathrm{~min}$. The resulting dissociation was monitored and incomplete. Thus, HSA was finally removed from the antibodies by injecting $100 \mathrm{mM}$ glycine- $\mathrm{HCl}(\mathrm{pH} 2.2)$ for $72 \mathrm{~s}$.

\subsection{Biacore experiments}

The experiments with the Biacore T100 (GE Healthcare Europe $\mathrm{GmbH}$, Germany) were performed using a CM5 sensor chip at $30^{\circ} \mathrm{C}$. Levels of protein bound to the surface were measured in resonance units (RU), where 1 RU roughly corresponded to a surface concentration of $1 \mathrm{pg} \mathrm{mm}^{-2}$ (Holford et al., 2012). The raw data were evaluated using BIAevaluation software 2.0.2 and Microsoft Excel.

\subsubsection{Immobilization of the antibodies}

The streptavidin molecules were immobilized on the CM5 chip using the amine coupling kit according to the manufacturer's instructions, and HBS-EP buffer (GE Healthcare Europe $\mathrm{GmbH}$, Germany) as the running dielectric. The streptavidin molecules were dissolved in $10 \mathrm{mM}$ sodium acetate ( $\mathrm{pH}$ 4.5). Initially, the carboxylated surface was activated for 7 min by injecting EDC/NHS. Pulses of a $0.93 \mu \mathrm{M}$ solution of streptavidin were then injected until a maximum immobilization level of $2747 \mathrm{RU}$ was reached. The remaining active groups were blocked with the injection of ethanolamine- $\mathrm{HCl}$ for $7 \mathrm{~min}$. The HSA-specific antibody was injected until saturation (1910 RU). All signals presented here are raw signal measurements minus signals recorded from blank injections and injections with a reference surface prepared by immobilizing biotinylated BrdU-specific antibodies on the streptavidin layer to a surface concentration equivalent to $700 \mathrm{RU}$.

\subsubsection{HSA measurement}

HSA samples were diluted in TBST to the desired concentrations and then injected onto the antibody-modified surface for $10 \mathrm{~min}$. The degree of binding was determined by measuring the SPR signal at the end of this association phase, which was followed by injecting TBST. The resulting dissociation was monitored and incomplete. Thus, HSA was finally removed from the antibodies by injecting $10 \mathrm{mM}$ glycine- $\mathrm{HCl}(\mathrm{pH} 1.5)$ for $72 \mathrm{~s}$.

\section{Results and discussion}

\subsection{Assay development using the li SPR system}

Amine coupling is one of the most frequently used methods for immobilizing receptors in biosensors (Homola, 2008; Jung et al., 2008), especially proteins, since most proteins contain available primary amine groups. The procedure starts with activation of the sensor surface using a mixture of EDC and NHS. The product is a reactive succinimide ester, which reacts spontaneously with the amine group of the ligand. When the desired immobilization level is reached, a solution of ethanolamine is injected to wash away non-covalently bound molecules and deactivate the unreacted NHS-ester (Löfås and Johnsson, 1990).

To immobilize streptavidin at high densities, a high concentration near the active surface is required. This can be achieved by optimizing electrostatic interactions between the negatively charged carboxylated surface and the ligand. Ligands are commonly dissolved in $10 \mathrm{mM}$ sodium acetate to maintain low ionic strength (Drescher et al., 2009; Johnsson et al., 1991). The $\mathrm{pH}$ of this solution should be at least one $\mathrm{pH}$ unit below the ligand's pI (isoelectric point) to ensure that the ligand is positively charged and thus present at high concentrations at the surface. However, the amine coupling procedure exploits uncharged amine groups, which prevail at high pH (Johnsson et al., 1991). Thus, the optimal pH for immobilization is always a compromise, and should be identified by $\mathrm{pH}$ scouting.

Therefore, solutions of streptavidin (pI 5-6; Diamandis and Christopoulos, 1991) in $10 \mathrm{mM}$ sodium acetate at several $\mathrm{pH}$ values were prepared and incubated with the nonactivated carboxylated surface (Fig. 1). The responses increased with increasing $\mathrm{pH}$ up to 4.5 , then decreased. Thus, $\mathrm{pH} 4.5$ was identified as optimal for the streptavidin immobilization. Under these conditions, streptavidin could be immobilized at densities equivalent to $3437 \mathrm{RU}$, somewhat lower than the densities detected during $\mathrm{pH}$ scouting (see Fig. 1). As mentioned above, non-covalently bound molecules were washed away by the ethanolamine injection during the immobilization procedure.

The surface concentration of the biotinylated antibody (2665 RU) was kept as high as possible to work under mass transport limitations to allow concentration-dependent binding (Fägerstam et al., 1992). The specificity of the antibody has been previously demonstrated in experiments, including controls with BSA (bovine serum albumin), which has $76 \%$ sequence homology with HSA (Henseleit et al., 2014). Furthermore, we performed regeneration scouting to identify optimal regeneration conditions, in which the antibodies' binding efficiency remained constant during several binding cycles (data not shown).

HSA concentrations ranging from barely detectable up to near saturation ( 0.4 to $192.5 \mathrm{nM})$ were injected sequentially onto the sensor surface (Fig. 2). 


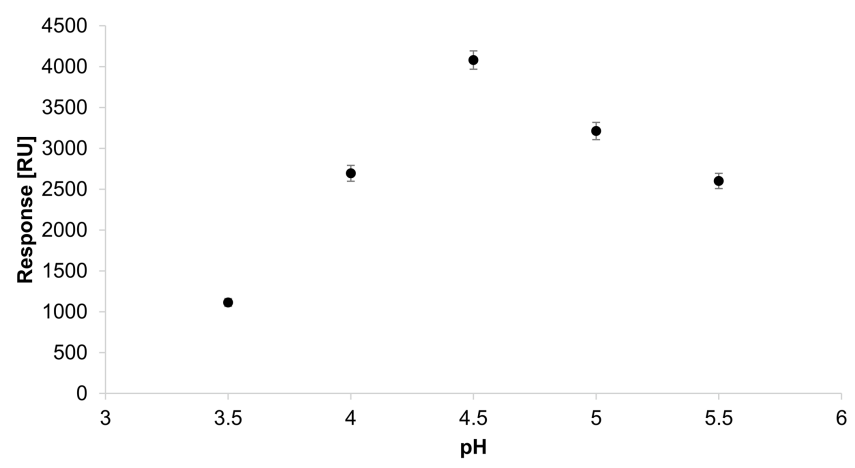

Figure 1. li SPR system responses of the electrostatically adsorbed streptavidin molecules vs. $\mathrm{pH}$. The data represent mean values of 1280 monitored spots across a $(9 \times 0.8) \mathrm{mm}^{2}$ measuring area; error bars indicate the corresponding standard deviations.

Deviation of the signals from the mean value resulting from triplicate analysis of 0.4 and $0.8 \mathrm{nM}$ HSA were substantial: 21 and $29 \%$, respectively. This is not surprising, as the limit of detection (LOD), calculated following recommendations by the International Union of Pure and Applied Chemistry (IUPAC) as 3 times the machine noise (determined as the deviation of signals resulting from three blank injections), was $30 \mathrm{RU}$, corresponding to $0.4 \mathrm{nM}$ HSA. The deviation from the mean value decreased with increases in HSA concentration (192.5 $\mathrm{nM}$ up to $7 \%$ ).

A typical response curve was recorded (Fig. 2, left panel), including a log-linear phase for HSA concentrations ranging from 0.8 to $48 \mathrm{nM}$, fitting the regression equation $\Delta \mathrm{RU}=60.517 \times \ln [\mathrm{HSA}]+66.97$ with a correlation coefficient $\left(R^{2}\right)$ of 0.9929 .

\section{2 lisPR vs. Biacore sensitivity}

To assess the li SPR system's utility for measuring HSA concentrations of in vitro hepatocyte cultures, we obtained comparative measurements using the Biacore platform. The results show that the immobilization level was lower on the

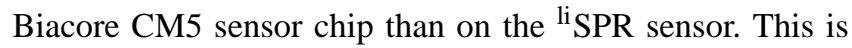
because the gold surface of the CM5 chip is functionalized with a hydrophilic hydroxyalkylthiol SAM, modified with a matrix of carboxymethylated dextran (Löfås and Johnsson, 1990). The carboxymethylated dextran matrix forms a protective layer (ca. $100 \mathrm{~nm}$, depending on $\mathrm{pH}$ and ionic strength) between the biomolecules and gold surface that is almost 50 times thicker than the SAM formed in the ${ }^{\text {li }}$ SPR system. This leads to signal masking due to evanescent waves decaying as a function of the distance from the gold surface (Frederix et al., 2003). Furthermore, the three-dimensional structure increases steric hindrance during the diffusion of the proteins inside the matrix.

HSA solutions with concentrations ranging from $0.2 \mathrm{nM}$ to $27.1 \mu \mathrm{M}$ were sequentially injected into the Biacore instru- ment, in duplicates, and a log-linear calibration curve ranging from $15 \mathrm{nM}$ to $9 \mu \mathrm{M}$ was obtained, fitting the regression equation $\Delta \mathrm{RU}=33.368 \times \ln [\mathrm{HSA}]-55.394$ with a correlation coefficient $\left(R^{2}\right)$ of 0.9992 .

Like the ${ }^{\text {li }}$ SPR measurements, the deviation of the signals from the mean value decreased, from 24 to $2 \%$, with increasing HSA concentrations.

As a further control, risks of false-positive results were minimized by subtracting signals arising from the reference surface (constructed to be as similar as possible to the sensing surface in the immunoassay by immobilizing unrelated antibodies via streptavidin) from the binding signals. Signals obtained from injecting $1.5 \mu \mathrm{M}$ HSA onto both the reference and measurement surfaces (showing that non-specific binding of HSA and the sensor surface was close to zero) are presented in Fig. 3. The fluctuations of the signals resulting from the lisPR system are due to effects of the samples being pumped backwards and forwards over the sensor surface to enhance contact time without wasting sample solution.

Figure 4 depicts the calibration curves obtained from the two systems in relation to HSA concentrations relevant to in vitro cultivated hepatocytes. In the multi-organ-chip system presented by Wagner et al., HSA concentrations reportedly range from 88 to $170 \mathrm{nM}$ HSA for purely hepatocyte cultures and from 3.3 to $3.8 \mathrm{nM}$ HSA for co-cultivated skin and liver tissue cultures (Wagner et al., 2013). Only $40 \%$ of the total on-chip volume is renewed every $12 \mathrm{~h}$ during the first 7 days and every $24 \mathrm{~h}$ during the next 7 days. Thus, HSA accumulates during the cultivation period.

Using the Biacore T100 in conjunction with our SPRbased immunoassay, the HSA concentrations in pure hepatocyte cultures could be detected easily by the presented immunoassay, but additional steps would be required to enhance signals sufficiently to monitor them in co-cultures, with accompanying increases in analysis time and costs.

In contrast, using the li SPR system in conjunction with our SPR-based immunoassay, HSA concentrations ranging from 0.8 to $48 \mathrm{nM}$ can be measured. Thus, samples from both types of cultures can be diluted, if necessary, for HSA determination. The system requires ca. $60 \mu \mathrm{L}$ samples. Hence, the presented results indicate that $\leq 14.6 \mu \mathrm{L}$ samples from co-cultivated liver and skin cells, and $\leq 0.6 \mu \mathrm{L}$ samples from pure hepatocyte cultures, are needed.

Furthermore, dilution offers the possibility of adding surfactants like Tween ${ }^{\circledR} 20$ to the samples to minimize the nonspecific binding of interfering sample components, which may occur by replacement of water molecules on the sensor surface by proteins (Vogler, 2012). Thus, adsorption is more likely on hydrophobic surfaces (with a water contact angle greater than $65^{\circ}$ ). SAMs are applied to form hydrophilic protective layers (Jans et al., 2008; Kausaite-Minkstimiene et al., 2010). However, gaps in these layers due to contaminants, temperature effects or incomplete self-assembling cannot be excluded (Love et al., 2005). Tween ${ }^{\circledR} 20$ is a nonionic molecule consisting of a hydrophilic head group and 

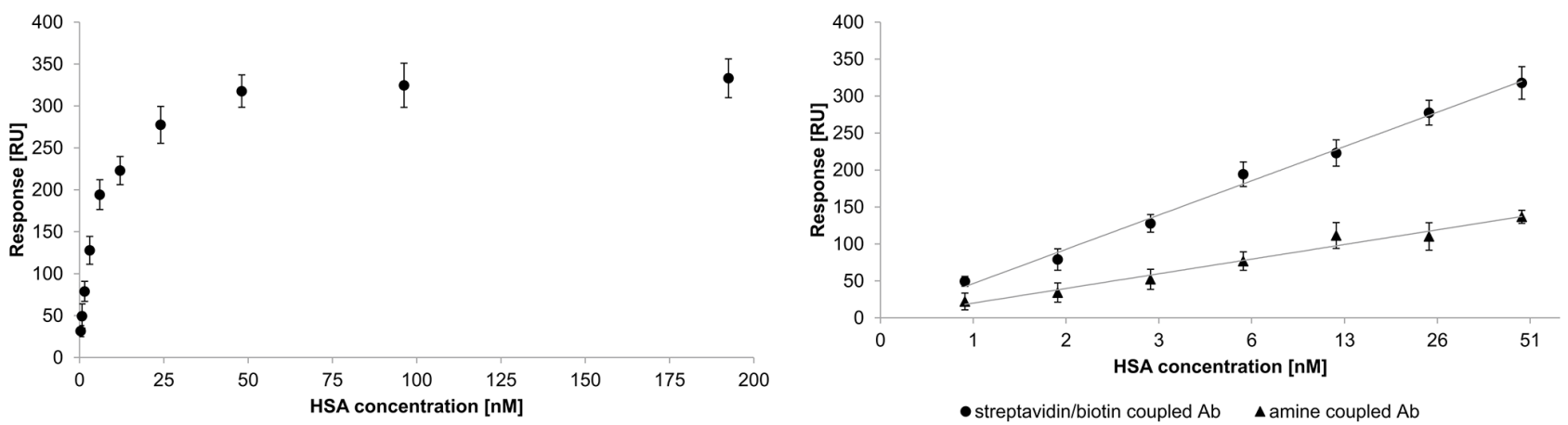

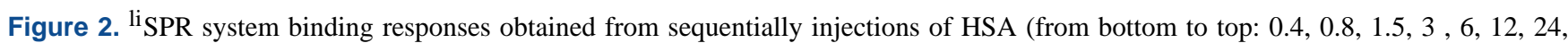
48, 96 and $192.5 \mathrm{nM}$; left panel) and the resulting calibration curve, with a correlation coefficient of 0.9929, obtained from trails with HSA concentrations ranging from 0.8 to $48 \mathrm{nM}$ (right panel). Signals arising from reference antibodies were subtracted from those arising from the HSA-specific antibodies. Error bars indicate the deviations of means from four replicates.
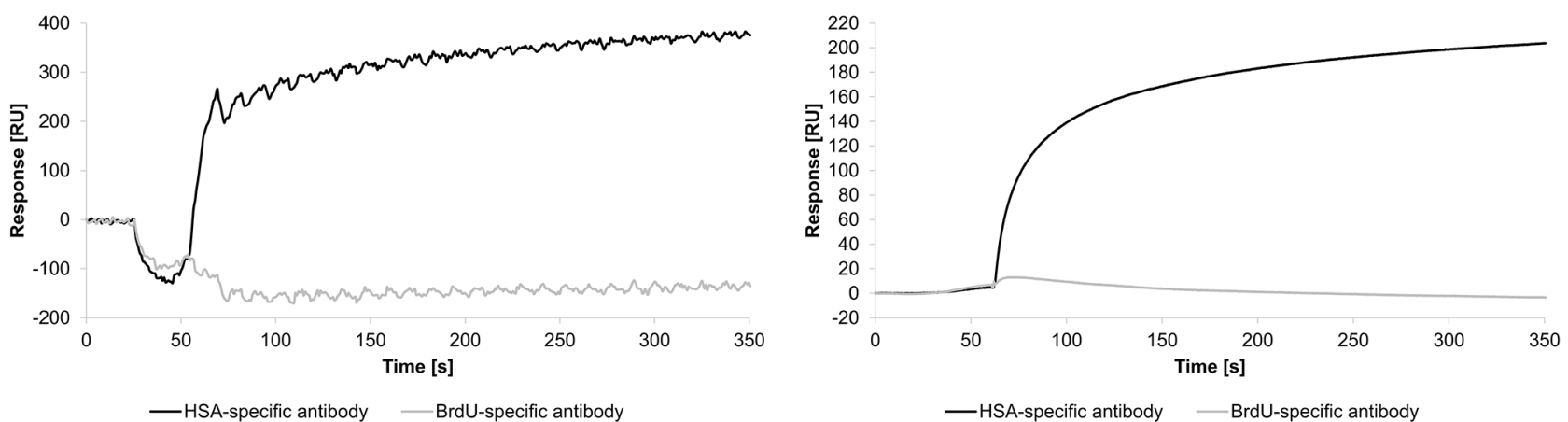

Figure 3. Sensorgrams showing the interaction of $1.5 \mu \mathrm{M}$ HSA with immobilized HSA-specific antibodies and BrdU-specific antibodies. The left and right panels depict the signals obtained using the ${ }^{\text {li }}$ SPR and Biacore T100 systems, respectively.

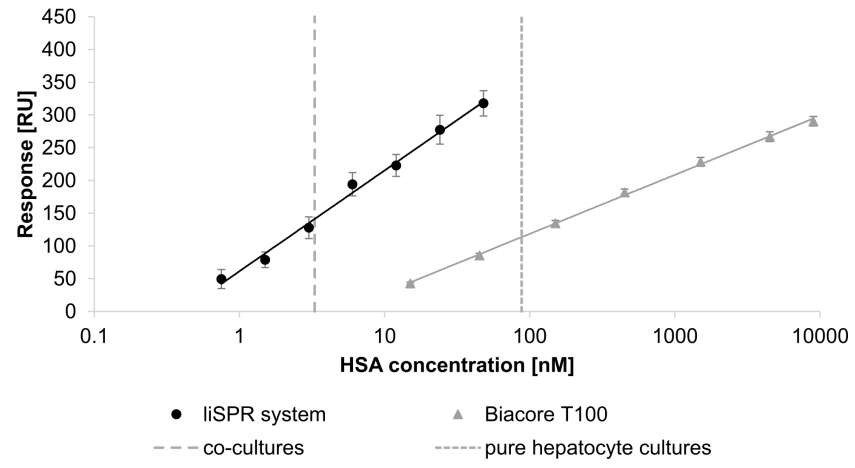

Figure 4. Comparison of the calibration curves obtained with the ${ }^{l i}$ SPR system and Biacore T100 in relation to the minimal HSA concentrations reportedly detected in co-cultivated and pure hepatocyte cultures in the multi-organ chip presented by Wagner et al. (2013). The signals arising from the reference antibodies were subtracted from those arising from the HSA-specific antibodies. Error bars indicate the deviations of means obtained from four replicates using

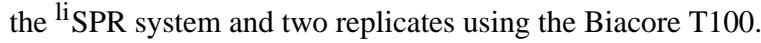

a hydrophobic tail (Kerwin, 2008) that suppresses nonspecific binding of proteins, by binding to the hydrophobic areas of the sensitive surface, thus displaying its hydrophilic head group to the samples.

\section{Conclusions}

Surface plasmon resonance (SPR) spectroscopy is being increasingly widely used in optical biosensors for detecting diverse clinical, food safety and environmental markers. SPR biosensors are also highly suitable for controlling biotechnological processes, due to their high sensitivity, rapidity and capacity for parallel measurements (which allows the use of control and reference surfaces to avoid false-positive results due to interfering sample components in simultaneous, realtime analyses).

Here, we present an SPR-based immunoassay for specific detection of HSA, which we tested in comparative trials with both the well-known Biacore platform and the cheaper bench-top liSPR system. The results show that the assay is capable of monitoring in vitro cultivated hepatocytes using

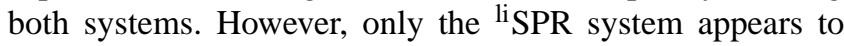


be capable of monitoring HSA levels in co-cultivated liver and skin cells, which may be as low as $3.3 \mathrm{nM}$ in samples with very small volumes (Wagner et al., 2013). Using the ${ }^{\text {li }}$ SPR system and our (re-usable) sensor surface, we obtained a log-linear calibration curve from 0.8 to $48 \mathrm{nM}$ HSA, theoretically allowing determinations of HSA in just $14.6 \mu \mathrm{L}$ samples from co-cultivated liver and skin cells, and $0.6 \mu \mathrm{L}$ samples from purely hepatocyte cultures.

In future work, we aim to measure HSA concentrations in effluents from hepatocyte cultures in the multi-organ chip presented by Wagner et al. (2013) to test the true potential of the developed immunoassay for monitoring liver cells.

Author contributions. Anja Henseleit, Elke Boschke and Thomas Bley designed the experiments and Carolin Pohl and Anja Henseleit carried them out. Anja Henseleit and Carolin Pohl analyzed the data. Anja Henseleit prepared the manuscript with contributions from all co-authors.

Acknowledgements. The authors thank D. Scharnweber and V. Hintze of the Institute of Materials Science TU Dresden for the opportunity to perform the Biacore experiments. We acknowledge the valuable advice on data interpretation provided by U. H. Yildiz of the Izmir Institute of Technology Department of Chemistry, Turkey, and both J. Homola and M. Bocková of the Institute of Photonics and Electronics AS, Czech Republic. We also thank E.-M. Materne of the Institute of Biotechnology TU Berlin and F. Sonntag of the Fraunhofer Institute for Material and Beam Technology (IWS) for useful discussions regarding the multi-organ-chip platform. This work was financially supported by the European Union and the Free State of Saxony (SAB project UNILOC).

Edited by: C.-D. Kohl

Reviewed by: two anonymous referees

\section{References}

Cao, C., Kim, J. P., Kim, B. W., Chae, H., Yoon, H. C., Yang, S. S., and Sim, S. J.: A strategy for sensitivity and specificity enhancements in prostate specific antigen- $\alpha 1$-antichymotrypsin detection based on surface plasmon resonance, 21, 2106-2113, 2006.

Cass, A. E. G., Cass, T., and Ligler, F. S.: Immobilized Biomolecules in Analysis: A Practical Approach, Oxford University Press, 1998.

Curry, S., Brick, P., and Franks, N. P.: Fatty acid binding to human serum albumin: new insights from crystallographic studies, Biochim. Biophys. Acta, 1441, 131-140, 1999.

Diamandis, E. P. and Christopoulos, T. K.: The biotin-(strept)avidin system: principles and applications in biotechnology, Clin. Chem., 37, 625-636, 1991.

Drescher, D. G., Ramakrishnan, N. A., and Drescher, M. J.: Surface plasmon resonance (SPR) analysis of binding interactions of proteins in inner-ear sensory epithelia, Methods Mol. Biol., 493, 323-343, 2009.
Fägerstam, L. G., Frostell-Karlsson, ̊., Karlsson, R., Persson, B., and Rönnberg, I.: Biospecific interaction analysis using surface plasmon resonance detection applied to kinetic, binding site and concentration analysis, J. Chromatogr. A, 597, 397-410, 1992.

Frederix, F., Bonroy, K., Laureyn, W., Reekmans, G., Campitelli, A., Dehaen, W., and Maes, G.: Enhanced performance of an affinity biosensor interface based on mixed self-assembled monolayers of thiols on gold, Langmuir, 19, 4351-4357, 2003.

Henseleit, A., Schmieder, S., Bley, T., Sonntag, F., Schilling, N., Quenzel, P., Danz, N., Klotzbach, U., and Boschke, E.: A compact and rapid aptasensor platform based on surface plasmon resonance, Eng. Life Sci., 11, 573-579, 2011.

Henseleit, A., Stürmer, J., Pohl, C., Haustein, N., Sonntag, F., Bley, T., and Boschke, E.: Surface plasmon resonance based detection of human serum albumin as a marker for hepatocytes activity., Intell. Sens. Sens. Netw. Inf. Process. ISSNIP 2014 IEEE Ninth Int. Conf., 1-5, 2014.

Holford, T. R. J., Davis, F., and Higson, S. P. J.: Recent trends in antibody based sensors, Biosens. Bioelectron., 34, 12-24, 2012.

Holmberg, A., Blomstergren, A., Nord, O., Lukacs, M., Lundeberg, J., and Uhlén, M.: The biotin-streptavidin interaction can be reversibly broken using water at elevated temperatures, Electrophoresis, 26, 501-510, 2005.

Homola, J.: Surface plasmon resonance sensors for detection of chemical and biological species, Chem. Rev., 108, 462-493, 2008.

Jans, K., Bonroy, K., De Palma, R., Reekmans, G., Jans, H., Laureyn, W., Smet, M., Borghs, G., and Maes, G.: Stability of mixed PEO-thiol SAMs for biosensing applications, Langmuir ACS J. Surf. Colloids, 24, 3949-3954, 2008.

Johnsson, B., Löfås, S., and Lindquist, G.: Immobilization of proteins to a carboxymethyldextran-modified gold surface for biospecific interaction analysis in surface plasmon resonance sensors, Anal. Biochem., 198, 268-277, 1991.

Jung, Y., Jeong, J. Y., and Chung, B. H.: Recent advances in immobilization methods of antibodies on solid supports, The Analyst, 133, 697-701, 2008.

Kausaite-Minkstimiene, A., Ramanaviciene, A., Kirlyte, J., and Ramanavicius, A.: Comparative study of random and oriented antibody immobilization techniques on the binding capacity of immunosensor, Anal. Chem., 82, 6401-6408, 2010.

Kerwin, B. A.: Polysorbates 20 and 80 used in the formulation of protein biotherapeutics: structure and degradation pathways, J. Pharm. Sci., 97, 2924-2935, 2008.

Kretschmann, E. and Raether, H.: Radiative decay of nonradiative surface plasmons excited by light, Z. Naturforsch A., 23, 21352136, 1968.

Löfås, S. and Johnsson, B.: A novel hydrogel matrix on gold surfaces in surface plasmon resonance sensors for fast and efficient covalent immobilization of ligands, J. Chem. Soc. Chem. Commun., 21, 1526-1528, 1990.

Love, J. C., Estroff, L. A., Kriebel, J. K., Nuzzo, R. G., and Whitesides, G. M.: Self-assembled monolayers of thiolates on metals as a form of nanotechnology, Chem. Rev., 105, 1103-1169, 2005.

Mertig, M., Kick, A., Bonsch, M., Katzschner, B., Voigt, J., Sonntag, F., Schilling, N., Klotzbach, U., Danz, N., Begemann, S., Herr, A., and Jung, M.: A novel platform technology for the detection of genetic variations by surface plasmon resonance, in 2009 IEEE Sensors, 392-395, 2009. 
Peluso, P., Wilson, D. S., Do, D., Tran, H., Venkatasubbaiah, M., Quincy, D., Heidecker, B., Poindexter, K., Tolani, N., Phelan, M., Witte, K., Jung, L. S., Wagner, P., and Nock, S.: Optimizing antibody immobilization strategies for the construction of protein microarrays, Anal. Biochem., 312, 113-124, 2003.

Schneider, B. H., Dickinson, E. L., Vach, M. D., Hoijer, J. V., and Howard, L. V.: Highly sensitive optical chip immunoassays in human serum, Biosens. Bioelectron., 15, 13-22, 2000.

Vogler, E. A.: Protein adsorption in three dimensions, Biomaterials, 33, 1201-1237, 2012.
Wagner, I., Materne, E.-M., Brincker, S., Süßbier, U., Frädrich, C., Busek, M., Sonntag, F., Sakharov, D. A., Trushkin, E. V., Tonevitsky, A. G., Lauster, R., and Marx, U.: A dynamic multiorgan-chip for long-term cultivation and substance testing proven by $3 \mathrm{D}$ human liver and skin tissue co-culture, Lab. Chip, 13, 3538-3547, 2013.

Yang, M.-H., Jong, S.-B., Chung, T.-W., Huang, Y.-F., and Ty, Y.-C.: Quartz crystal microbalance in clinical application, in: Biosensors for Health, Environment and Biosecurity, edited by: Serra, P. A., InTech., 257-272, 2011. 